\title{
Intralipid Infusion is the Current Favorite of Gynecologists for Immunotherapy
}

\author{
Gautam N. Allahbadia ${ }^{1}$
}

Published online: 11 July 2015

(C) Federation of Obstetric \& Gynecological Societies of India 2015

\begin{abstract}
About the Author

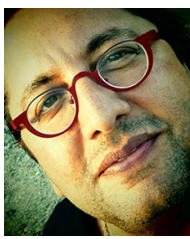

Gautam N. Allahbadia, MD, is the Editor-in-chief of the Journal of Obstetrics \& Gynecology of India as well as the IVF Lite (Journal of Minimal Stimulation IVF). He is the Medical Director of Rotunda-The Center for Human Reproduction, the world-renowned fertility clinic at Bandra, Mumbai, India as also the New Hope IVF Clinic at Sharjah, UAE. He is a noted world authority on Ultrasound-guided Embryo Transfers and one of the pioneers in Third Party Reproduction in SouthEast Asia. Dr. Allahbadia was responsible for India's first transethnic surrogate pregnancy involving a Chinese couple's baby delivered by an unrelated Indian surrogate mother. He has over 100 peer-reviewed publications to his credit and is on the Editorial Board of several International Journals. Throughout his career, Dr. Allahbadia has been instrumental in developing new fertility-enhancing protocols and propagating the use of Ultrasound in Embryo Transfer procedures. You can read more about his work at www.gautamallahbadia.com.
\end{abstract}

\section{Introduction}

Aspects of the immunological relationship between mother and conceptus still remain a mystery, although the recent advances in molecular biology have enlightened some of the parameters that participate in feto-maternal cross-talk during implantation. The atypical expression of major histocompatibility complex (MHC), the specific roles of some hormones and cytokines, as well as the temporal and

Gautam N Allahbadia is the Editor-in-chief of the Journal of Obstetrics \& Gynecology of India as well as the IVF Lite (Journal of Minimal Stimulation IVF) and Medical Director at Rotunda-The Center For Human Reproduction, Mumbai, India \& New Hope IVF, Sharjah, UAE.

Gautam N. Allahbadia

ivfwaladoc@gmail.com

1 Rotunda-The Center For Human Reproduction, Mumbai, India spatial distributions of uterine natural killer (uNK) cells, represent substantive parameters of feto-maternal immunotolerance during implantation [1]. Although human maternal and fetal immunology is difficult to investigate, aberrant immune responses and an imbalanced cytokine network may be related to infertility, implantation failures after IVF, and recurrent pregnancy losses [2]. Patients with recurrent implantation failure (RIF) should be tested for inherited and acquired thrombophilias. Each patient should be individually assessed and counseled regarding management with low-molecular-weight heparin (LMWH). Empirical treatment with LMWH, aspirin, or corticosteroids is not effective for women with RIF who have negative thrombophilic tests [3]. If thrombophilic tests are normal, patients should be tested for immunological causes. The findings of a recent study suggest that increases in the percentage of CD56(dim) cells and NK cytotoxicity in peripheral blood may be important contributing factors for both RSA and IVF failure [4]. Human leukocyte antigen (HLA)-DQA $1 * 0505$ sharing or the maternal killer 
immunoglobulin-like receptor (KIR) repertoire is associated with recurrent spontaneous abortion (RSA) or repeated implantation failure (RIF) [5] and if abnormal, the patient might then benefit from intravenous immunoglobulin (IVIg) therapy [3]. IVIg has been successful in the treatment of recurrent miscarriage and recurrent implantation failure among women with elevated anti-phospholipid antibodies (APA) and/or NK cell activity [6]. When the pregnancy outcomes of women with a history of reproductive failure and elevated NK cell cytotoxicity treated with intralipid were compared with women treated with IVIg, no differences were seen [6]. Side-by-side comparison showed that synthetic pre-implantation factor (sPIF) is equally effective to inhibit NK cell toxicity at a lower dose than intravenous gamma immunoglobulin or intralipid treatment currently used [7]. sPIF is not yet available commercially, but intralipid infusions are available globally. Intralipid (IL) is a synthetic product composed of $10 \%$ soybean oil, $1,2 \%$ egg yolk phospholipids, $2.25 \%$ glycerin, and water. When indicated, IL is infused 7-10 days prior to embryo transfer (ET), and one more time again after a positive pregnancy in women whose $\mathrm{NKa}$ is due to an autoimmune causes (antiphospholipid antibodies and/or antithyroid antibodies) [8]. In cases of alloimmune implantation dysfunction (DQa and/HLA matching between the embryo recipient and the male partner), the same applies, but in this situation, the infusion is repeated at 2-4 week intervals until the 24th week of pregnancy [8]. IL costs about 10 times less than IVIg, is not a blood product, and is without significant side effects [8].

\section{Discussion}

Embryo implantation and formation of a functional placenta are complex processes that require a plethora of regulatory mechanisms involving both maternal and embryonic cells. Recently, an important role in this complicated cells and factors network was assigned to the decidual stromal cells (DSCs) and trophoblast cells. Decidualization includes biochemical changes that trigger DSCs to produce a number of factors required for the implantation and induction of immunotolerance in the maternal immune system [9]. Immunotolerance is achieved by a cascade of strictly controlled events starting with selective homing of immune cells to the feto-maternal site, regulated proliferation, and predominant differentiation into a regulatory type of immune cells. Furthermore, cytotoxic effector functions are reduced owing to the influence of steroid hormones, factors, cytokines, and inhibitory receptors. Altogether the entire immune system of the mother is switched to tolerogenic functional state, which is a prerequisite for the successful maintenance of pregnancy [9]. Natural killer (NK) cells are a type of lymphocyte circulating in peripheral blood named because of their effector functions in killing target cells. Immune cells that share similar phenotypic characteristics but are poor killers populate the uterine lining at implantation and during early pregnancy when the placenta is established. The functions of these uterine NK (uNK) cells are essentially unknown but available data point to a role in regulating placentation in concert with other elements of the decidua and invading trophoblast cells [1].

During implantation, maternal immunoactivation and tolerance are not only limited to the decidua but are also observed in the periphery, predominantly affecting the innate immune system [1,9]. Since unexplained female infertility, as well as recurrent spontaneous abortion and implantation failure, is thought to be associated with pathological maternal immunotolerance mechanisms, Miko et al.'s study [10] focused on immune profile analysis of IVF candidates. Previous studies on peripheral NK cell characteristics of IVF patients have been limited to the comparison of blood samples taken prior to the IVF procedure. Miko et al. performed a follow-up study and compared patient's data obtained on the day of oocyte collection with the data collected 1 week after embryo transfer [10]. The aim was to investigate phenotypic (subpopulations, CD69, T cell immunoglobulin mucin 3 and NK-activating receptor expression), and functional (perforin and CD107a expression) changes in the peripheral NK and NK T (NKT)-like cell populations. During this short period of time around the IVF procedure, women with failed IVF reflected unfavorable Th1-oriented changes of NK and NKT-like cells. In comparison the follow-up data for women with successful conception remained principally constant [10].

All immunotherapies essentially target the uNK and/or peripheral blood NK cells in women with recurrent pregnancy failure. The story began in the late 1990s and early 2000s with paternal lymphocyte immunization therapy (LIT). Twelve patients with recurrent miscarriage were treated with immunization with paternal lymphocyte vaccinations three times over a period of 12-14 weeks [11]. Before and 2 weeks after the final inoculation, lymphocyte subsets and intracellular interferon (IFN)-gamma and/or interleukin (IL)-4 production were examined by flow cytometry [11]. Seven of nine patients who exhibited remarkable decreases in $\mathrm{Th} 1 / \mathrm{Th} 2$ ratios became pregnant within 6 months after three courses of immunotherapy, and four women delivered healthy babies, while none of the three patients who exhibited an increased or unchanged Th1/Th2 ratio had full-term pregnancies (chi2 $<0.0001$ ). The study findings suggested that a shift of Th1-dominant to Th2dominant status by vaccination might play important roles in maintaining successful pregnancies [11]. 
Safe, effective, and inexpensive alternatives to partner LIT are being sought. IVIg appears effective, but is expensive. A published double-blinded randomized controlled trial in which Intralipid (Kabi Vitrum, Toronto, Ontario) was used as a control versus trophoblast membrane vesicles was reviewed by Clark [12]. A prediction made from those data was then tested using the DBA/2mated $\mathrm{CBA} / \mathrm{J}$ mouse model of recurrent spontaneous abortion. He hypothesized from the human clinical trial data that Intralipid even in small doses could be an effective anti-abortion treatment [12]. Intralipid was highly effective in preventing abortion in mice, and protection was prolonged. This may be explained by previous data in the literature showing that Intralipid affects the reticuloendothelial system of the recipient [13]. Clark summarized that Intralipid might be an effective treatment for human recurrent miscarriages and a comparison of intralipid to partner LIT or IVIg would be worthwhile [12].

Roussev et al. [14] compared the abilities of intravenous immunoglobulin (IVIg), intralipid, and soluble human leukocyte antigen (sHLA)-G to suppress NK cell cytotoxicity in an in vitro assay. Blood samples taken from 275 women experiencing reproductive failure were analyzed for NK cytotoxicity and the suppression of NK cytotoxicity by IVIg 4 and $2 \mathrm{mg} / \mathrm{mL}$ $(n=275)$; intralipid 18 and $9 \mathrm{mg} / \mathrm{mL}(n=275)$; and sHLA-G 70 and $35 \mathrm{ng} / \mathrm{mL}$ ( $n=50)$, using immunofluorescent-labeled K562 cells as targets and flow cytometry. NK cytotoxicity was suppressed in all samples. Among patients with normal NK cell activity, IVIg suppressed NK cytotoxicity by $44.9 \pm 8.1 \%$, intralipid suppressed NK killing by $45.2 \pm 8.3 \%$, and sHLA$\mathrm{G}$ suppressed it by $49.0 \pm 9.2 \%$. When specimens with abnormal NK activity were observed for suppression of cytotoxicity, IVIg suppressed by $38.9 \pm 5.4 \%$, intralipid suppressed by $39.8 \pm 6.2 \%$, and sHLA-G suppressed by $39.9 \pm 5.0 \%$. The study summarized that IVIg, intralipid, and sHLA-G suppressed NK cell cytotoxicity with equal efficacy in an in vitro assay [14].

In vitro investigations have revealed the ability of intralipids to suppress NK cytotoxicity [14]. Evidence from both animal and human studies suggests that intralipid administered intravenously may enhance implantation and maintenance of pregnancy when the patient has an abnormal NK cell level or function [12]. The aim of an American study was to establish the duration and efficacy of intralipids' suppressive effect on NK cell functional activity [15]. Fifty patients with abnormal NK activity (NKa) results received intralipid $20 \%$ i.v. $(9 \mathrm{mg} / \mathrm{mL}$ total blood volume - corresponding to $2 \mathrm{~mL}$ of intralipid $20 \%$ diluted in $250 \mathrm{~mL}$ saline; or $18 \mathrm{mg} / \mathrm{mL}-$ corresponding to $4 \mathrm{~mL}$ of intralipid $20 \%$ diluted in $250 \mathrm{~mL}$ saline) infusions and their NKa were tested periodically. The determination of NK cell function was performed by flow cytometry using K562 cells as targets. Fifty women with abnormal NKa-testing received intralipid infusions. 39
(78 \%) showed NKa suppression within the normal range the first week after infusion, 11 (22\%), showed suppression, but still above the normal threshold. They received second infusion 2-3 weeks later. In 10 patients, the Nka activity was normalized the following week. Four patients had three intralipid infusions in 2-week periods in between, and after the third infusion, all showed normal NKa activity. In 47 patients, the suppressive effect of the Intralipid after the normalization of NKa lasted between 6 and 9 weeks; in two patients, this benefit lasted 5 weeks; and in one patient, the effect lasted 4 weeks. The authors concluded that intralipid therapy is effective in suppressing in vivo abnormal NK cell functional activity [15]. Their results suggested that intralipids can be used successfully as a therapeutic option to modulate abnormal NK activity in women with reproductive failure [15].

A search of PubMed was made to identify the published data regarding diagnosis and treatment of reproductive failure [6]. Results were compared with the frequencies of APA in 2995 women with histories of unexplained infertility, recurrent implantation failure, recurrent pregnancy loss, and fertile women. In addition, pregnancy outcomes among 442 women experiencing reproductive failure and elevated NK cell activity after treatment with IVIg $(N=242)$ or intralipids $(N=200)$ were compared. The prevalence of APA was the same among women with the diagnosis of unexplained infertility, recurrent implantation failure, and recurrent miscarriage. Heparin and aspirin are successful in the treatment of elevated APA among women with recurrent miscarriage but not with recurrent implantation failure. IVIg was successful in the treatment of recurrent miscarriage and recurrent implantation failure among women with elevated APA and/or NK cell activity. When the pregnancy outcomes of women with a history of reproductive failure and elevated NK cell cytotoxicity treated with intralipid were compared with women treated with IVIg, no differences were seen [6]. The authors concluded by stating that immunotherapy for treatment of reproductive failure enhances live birth but only in those women displaying abnormal immunologic risk factors [6].

On the other hand, Check [16] opined that there are no immunological studies that can determine if a woman needs immune suppression. The best way to decide is the history; the more the miscarriages without any other identifiable cause the more likely that the passive immunotherapy may be helpful. If intralipid proves as efficacious as IVIg, it will be a lot less expensive [16].

\section{Recent Advances}

Embryo-secreted preimplantation factor (PIF) is necessary for, and its concentration correlates with, embryo development in humans by promoting implantation and trophoblast invasion [7]. Synthetic PIF (sPIF) modulates 
systemic immunity and is effective in autoimmune disease models [17]. sPIF binds monocytes and activated T and B cells, leading to immune tolerance without suppression. A recent study examined the effect of SPIF on NK cell cytotoxicity in 107 consecutive nonselected, nonpregnant patients with recurrent pregnancy loss (RPL) and 26 infertile IVF patients (controls) [7]. The effects of sPIF, intravenous gamma immunoglobulin (IVIg), intralipid, and scrambled PIF (PIFscr; negative control) on NK cell cytotoxicity to peripheral-blood cells were compared by flow cytometry. In patients with RPL, sPIF inhibited NK cell cytotoxicity at doses of 2.5 and $25 \mathrm{ng} / \mathrm{ml}$ (37 and $42 \%)$ compared with PIFscr $(18 \% ; P<0.001)$. Pre-incubation of blood from infertile patients with SPIF for $24 \mathrm{~h}$ decreased NKCD69 + expression versus incubation with PIFscr $(P<0.05)$. The authors concluded that SPIF inhibits NK cell cytotoxicity by reducing NKCD69 expression, suggesting its significant role in RPL patients [7]. There is a continuous search to identify safe and effective agents to counteract recurrent pregnancy loss (RPL). Preimplantation factor (PIF) secreted by the embryo at the two-cell stage is present throughout viable pregnancy but absent in nonviable pregnancy [7, 17]. Its immunomodulatory (not suppressive) effects promote embryo acceptance and maintenance by mother/host, control inflammation, facilitate uterine environment, and placental embedding [7, 17]. Synthetic PIF (sPIF) was used to complete PIF's role as a targeted, safe treatment for immune-based RPL. Previous reports showed sPIF's significant protective systemic effect against maternal factors present in RPL serum [18]. The current study examined sPIF's ability to inhibit the local protective toxicity induced by NK immune cells in a representative number of RPL patients. When elevated in blood, NK cells are associated with RPL. Low-dose physiological sPIF was highly effective to inhibit NK cell toxicity. Side-by-side comparison showed that sPIF is equally effective at a lower dose than intravenous gamma immunoglobulin or IL treatment currently used [7]. The sPIF effect on NK cells was targeted, indicating specific action. Overall, sPIF may represent a safe, effective, and nontoxic immune-based therapy against RPL. Until we get sPIF commercially, intralipid as the Gynecologist's favorite remains in 2015 .

\section{Conclusion}

In vitro investigations have revealed the ability of intralipids to suppress NK cytotoxicity. Evidences from both animal and human studies suggest that intralipid administered intravenously may enhance implantation and maintenance of pregnancy when the patient has an abnormal NK cell level or function. However, there is insufficient current evidence of acceptable quality to permit an assumption that intralipid therapy is an effective treatment for women undergoing repeated implantation failures. Shreeve and Sadek [19] in their elegant review have concluded that appropriately controlled, large-scale, confirmatory studies are necessary to prove the efficacy of intralipid before it can be recommended for routine use.

Conflict of interest None.

\section{References}

1. Oreshkova T, Dimitrov R, Mourdjeva M. A cross-talk of decidual stromal cells, trophoblast, and immune cells: a prerequisite for the success of pregnancy. Am J Reprod Immunol. 2012;68(5):366-73. doi:10.1111/j.1600-0897.2012.01165.x.

2. Makrigiannakis A, Petsas G, Toth B, et al. Recent advances in understanding immunology of reproductive failure. J Reprod Immunol. 2011;90(1):96-104. doi:10.1016/j.jri.2011.03.006.

3. Fatemi H, Popovic-Todorovic B. Implantation in assisted reproduction: a look at endometrial receptivity. Reprod Biomed Online. 2013;27(5):530-8. doi:10.1016/j.rbmo.2013.05.018.

4. Karami N, Boroujerdnia MG, Nikbakht R, et al. Enhancement of peripheral blood CD56(dim) cell and NK cell cytotoxicity in women with recurrent spontaneous abortion or in vitro fertilization failure. J Reprod Immunol. 2012;95(1-2):87-92. doi: 10.1016/j.jri.2012.06.005.

5. Varla-Leftherioti M, Keramitsoglou T, Parapanissiou E, et al. HLA-DQA $1 * 0505$ sharing and killer immunoglobulin-like receptors in sub fertilecouples: report from the 15th International Histocompatibility Workshop. Tissue Antigens. 2010;75(6):668-72. doi:10.1111/j.1399-0039.2010.01451.x.Epub.

6. Coulam CB, Acacio B. Does immunotherapy for treatment of reproductive failure enhance live births? Am J Reprod Immunol. 2012;67(4):296-304. doi:10.1111/j.1600-0897.2012.01111.x.

7. Roussev RG, Dons'koi BV, Stamatkin C, et al. Preimplantation factor inhibits circulating natural killer cell cytotoxicity and reduces CD69 expression: implications for recurrent pregnancy loss therapy. Reprod Biomed Online. 2013;26(1):79-87. doi: 10.1016/j.rbmo.2012.09.017.

8. CARE Fertility Forum Index. Intralipids - All you need to know. http://www.carefertility.com/ivf/viewtopic.php?t=39200. Accessed 6 June 2015

9. Blanco O, Leno-Durán E, Morales JC, et al. Human decidual stromal cells protect lymphocytes from apoptosis. Placenta. 2009;30(8):677-85. doi:10.1016/j.placenta.2009.05.011.

10. Miko E, Manfai Z, Meggyes M, et al. Possible role of natural killer and natural killer T-like cells in implantation failure after IVF. Reprod Biomed Online. 2010;21(6):750-6. doi:10.1016/ j.rbmo.2010.07.012.

11. Hayakawa S, Karasaki-Suzuki M, Itoh T, et al. Effects of paternal lymphocyte immunization on peripheral Th1/Th2 balance and TCR $\mathrm{V}$ beta and $\mathrm{V}$ gamma repertoire usage of patients with recurrent spontaneous abortions. Am J Reprod Immunol. 2000;43(2):107-15.

12. Clark DA. Intralipid as treatment for recurrent unexplained abortion? Am J Reprod Immunol. 1994;32(4):290-3.

13. Appelgren LE, Rössner S. Tissue distribution of 14C-labelled triolein in a soybean oil emulsion. An autoradiographic study in rat and mouse. Nutr Metab. 1980;24(2):122-34.

14. Roussev RG, Ng SC, Coulam CB. Natural killer cell functional activity suppression by intravenous immunoglobulin, intralipid 
and soluble human leukocyte antigen-G. Am J Reprod Immunol. 2007;57(4):262-9.

15. Roussev RG, Acacio B, Ng SC, et al. Duration of intralipid's suppressive effect on NK cell's functional activity. Am J Reprod Immunol. 2008;60(3):258-63. doi:10.1111/j.1600-0897.2008.00621.x.

16. Check JH. A practical approach to the prevention of miscarriage: Part 3-Passive immunotherapy. Clin Exp Obstet Gynecol. 2010;37(2):81-3.

17. Barnea ER, Kirk D, Todorova K, et al. PIF direct immune regulation: blocks mitogen-activated PBMCs proliferation, promotes $\mathrm{TH} 2 / \mathrm{TH} 1$ bias, independent of $\mathrm{Ca}(2$.$) . Immunobiology.$ 2015;220(7):865-75. doi:10.1016/j.imbio.2015.01.010.
18. Barnea ER, Lubman DM, Liu YH, et al. Insight into PreImplantation Factor (PIF*) mechanism for embryo protection and development: target oxidative stress and protein misfolding (PDI and HSP) through essential RIPK binding site. PLoS One. 2014;9(7):e100263. doi:10.1371/journal.pone.0100263.

19. Shreeve N, Sadek K. Intralipid therapy for recurrent implantation failure: new hope or false dawn? J Reprod Immunol. 2012;93(1):38-40. doi:10.1016/j.jri.2011.11.003. 